\title{
Optimizing Transport Time from Accident to Hospital: When to Drive and When to Fly?
}

\author{
D. V. Weerheijm, ${ }^{1}$ M. H. Wieringa, ${ }^{2}$ J. Biert, ${ }^{3}$ and N. Hoogerwerf ${ }^{1,4}$ \\ ${ }^{1}$ Department of Anaesthesiology, Radboud University Nijmegen Medical Centre, P.O. Box 9101, 6500 HB Nijmegen, The Netherlands \\ ${ }^{2}$ Department of Otorhinolaryngology and Head and Neck surgery, Erasmus Medical Centre, Rotterdam, The Netherlands \\ ${ }^{3}$ Department of Surgery, University Nijmegen Medical Centre, Nijmegen, The Netherlands \\ ${ }^{4}$ Regional Emergency Healthcare Network, Radboud University Nijmegen Medical Centre, Nijmegen, The Netherlands
}

Correspondence should be addressed to N. Hoogerwerf, n.hoogerwerf@anes.umcn.nl

Received 30 August 2012; Accepted 20 September 2012

Academic Editors: C. C. Chang and R. Cirocchi

Copyright ( 2012 D. V. Weerheijm et al. This is an open access article distributed under the Creative Commons Attribution License, which permits unrestricted use, distribution, and reproduction in any medium, provided the original work is properly cited.

\begin{abstract}
Objective. In prehospital emergency medicine, rapid transport to a hospital is often required. Transport can be done by ambulance or HEMS (Helicopter Emergency Medical Service). Factors influencing transport time are mostly unknown. This study aims to identify these factors and optimize prehospital transport times. Methods. This is a retrospective study with HEMS data from 20102011. Trauma mechanism, patient characteristics, and weather conditions were collected from each transport. Distance to the hospital, predicted transport time, and the actual transport time were calculated. Each factor was analysed to determine influence on transport time. Results. We analysed 732 cases ( 612 by ambulance and 120 by helicopter) and found no association between mechanism of trauma, age, time of day, or weather conditions on transport time. However, transport by HEMS helicopter was nearly always faster, even at short distances. The average transport time of ambulance transport was 79\% (95\% CI 77.1-80.3) of target time, while in helicopter transport this was 39\% (95\% CI 37.4-42.9). Conclusion. This study shows the advantage of the HEMS helicopter in rapid transport from the scene to the hospital. Transport by helicopter was twice as fast as ground transport, independent of trauma mechanism, distance to hospital, or weather conditions.
\end{abstract}

\section{Introduction}

In prehospital emergency medicine, the importance of optimal use of the golden hour, the first hour after an accident, is commonly accepted. In the Netherlands, there is a good coverage of ground ambulance care with nurse EMT's, supported by four helicopter emergency medical services (HEMS) with a physician (either an anaesthesiologist or a trauma surgeon) on board. In the Netherlands these HEMS are called Mobile Medical Teams (MMT), and are complementary in the trauma care delivered by ambulances [1]. The distance to the nearest hospital is generally short but can be as high as 30-40 minutes when transport to a level 1 trauma centre is needed [2]. Transport to the hospital can be done by ambulance (ground transport) or by HEMS (air transport). Transport in an ambulance offers more options to perform medical procedures during transport, while transport by
HEMS reduces therapeutic options during transport. Also, the ambulance has no take-off time like the helicopter. Furthermore, not all hospitals have adequate landing facilities. In these cases the HEMS has to land at a nearby field and transport the patient to the hospital by ambulance after landing, losing precious time. Finally, during very bad weather the HEMS crew cannot use the helicopter (dense fog, heavy rain, or wind) and has to drive to the scene of accident. However, HEMS transport has several advantages: traffic density and accident location do not influence transport time, there are fewer acceleration and sudden deceleration movements, and transport to a distant level 1 trauma hospital can be achieved within acceptable time limits.

The advantage of transport to hospital by air transport in a densely populated, rural setting can be debated. Although we found some literature that the helicopter is faster than the ambulance when travelling to the scene of an accident 
$[3,4]$, we couldn't find conclusive evidence which factors determine transport speed for both air and ground transport to the hospital. The choice of transport method is often made by distance, and sometimes by severity of injury [5] but evidence based guidelines do not exist yet. Although the choice to transport by ambulance or by helicopter is not based on speed alone, it can be a decisive factor. This study aims to identify which factors contribute to transport time, so we can create a model which predicts transport time from any given location in the Netherlands to the hospital of choice for both ground and air transport.

\section{Methods}

2.1. Setting. We used data from the Nijmegen HEMS, which covers the south and eastern part of the Netherlands, a generally rural area. This HEMS is a 24/7 present team, which is dispatched to an incident by the regional ambulance dispatch centre.

Within the operating area of this HEMS are 5 trauma centres. Specialized burn clinics are not located in the regular operating area and burn patients have to be transported relatively far (approximately 100 kilometres). Local hospitals are readily available throughout the entire area, but do not offer definitive treatment for severe injuries and traumatic brain injury (TBI). Paediatric ICU care is only available in 3 of the 5 surrounding level 1 trauma centres. In some cases, the dispatch is for an incident outside the normal area, when additional trauma care is asked for by the surrounding dispatch centres or MMT's.

2.2. Design. This is a retrospective study. The Nijmegen HEMS uses an MS-Access database, in which the HEMS physician fills in an extensive amount of logistical and medical data after each scramble. We used data from this database over the period of 2010 and 2011. We extracted the following variables from this database: date, time, location of accident, location of landing, MIST-report of ambulance nurse, treatment report from the HEMS physician, Revised Trauma Score (RTS), and need for intubation.

Extracted data were imported in a custom made FileMaker Pro 11 database, which was used for further data collection. Using Google Maps, we calculated the fastest (not necessarily the shortest) ground route to the hospital in kilometres, and thus a target time in minutes. This calculated target time is the optimal travel time while respecting speed limits. Using this target time, we calculated a relative transport time (RTT), the actual measured transport time divided by the calculated target time. This is expressed as a percentage of the target time, for example, a relative transport time of $50 \%$ means the transport took half of the calculated target time.

For trauma mechanism, the following variables were dichotomously "yes or no" scored: high-energetic, traumatic brain injury, resuscitation on scene, need for intubation, child (age < 16 years), drowning, and burn wounds.

The weather conditions were difficult to obtain, because there is no accurate public available record of the exact weather condition at the moment of scramble. Therefore, we collected data from the national weather institute (KNMI), which are averages for amount of rain, presence of snow, wind direction, and temperature for that day.

The air distance is measured in a straight line from the scene of accident to the hospital, regardless of actual landing zone. We used the website http://www.afstandmeten.nl/ (which uses Google Maps data) to perform these measurements.

2.3. Primary Data Analysis. Initially all patients recorded in the Nijmegen HEMS database between 1-1-2010 and 31-122011 were included, but in case the physician did not accompany the patient to the hospital, this record was excluded.

We used SPSS statistics version 19 by IBM for statistical analysis. For analysis of occurrence of trauma mechanisms, we calculated the presence of the various trauma mechanisms within both transport methods (ambulance versus HEMS). Differences were tested using a Pearson's chi-square. To analyze the effect of the different trauma mechanisms on transport time we used an independent samples $t$ test. In a scatter plot relative transport time and absolute time benefit (in minutes) were plotted against distance. Because we have no helicopter transport data below 20 kilometre, and no ground ambulance data above 100 kilometres, we excluded data below and above this range for the scatter box analysis. A regression line for both transport methods was calculated using linear regression analysis. A $P$ value less than 0.05 was considered as significant.

\section{Results}

3.1. Patient Characteristics. Within the selected period 1394 scrambles (686 in 2010 and 708 in 2011) were recorded by the Nijmegen HEMS. Of these 634 were excluded because the physician did not accompany the patient to the hospital. Essential values were missing in 28 cases, leaving 732 cases in the analyses. A total of 612 patients were transported by ambulance (84\%) and 120 patients by HEMS (16\%). There is no significant difference between groups for physiological disturbance of the trauma patient as compared by Revised Trauma Score (RTS): mean RTS for ambulance: 9, 5, HEMS: $9,4, P=0.751$.

3.2. Transport Time. In Table 1 patient and trauma characteristics are shown. In case of air transport more patients were intubated prior to transport, compared to ground transport. Also, relatively more children and burn victims are transported by air. There were no differences between high energetic trauma and traumatic brain injury in transport method.

Table 2 shows that on average, HEMS transport was much faster than ambulance transport. The relative transport time for ambulance transport was $79 \%$ and for HEMS $39 \%$. The average absolute benefit in minutes on the target time was over 4 times higher for HEMS transport than for ambulance transport ( 7 versus 32 minutes). It should be noted though that HEMS transport was used at significantly longer average distances than ambulance transport, both when measured by road distance and air distance. 
TABle 1: Patient or trauma characteristics. Number (percentage) of patient characteristics per transport method.

\begin{tabular}{lccc}
\hline & $\begin{array}{c}\text { Total } \\
(n=733)\end{array}$ & $\begin{array}{c}\text { Ambulance } \\
(n=612)\end{array}$ & $\begin{array}{c}\text { HEMS } \\
(n=120)\end{array}$ \\
\hline High energy trauma & $483(34 \%)$ & $399(65 \%)$ & $84(70 \%)$ \\
Traumatic brain injury & $424(58 \%)$ & $346(57 \%)$ & $78(65 \%)$ \\
Intubation* & $447(61 \%)$ & $339(55 \%)$ & $108(90 \%)$ \\
Resuscitation & $72(10 \%)$ & $67(11 \%)$ & $6(5.0 \%)$ \\
Child (age $<16$ years) & $114(16 \%)$ & $86(14 \%)$ & $28(23 \%)$ \\
Drowning & $21(2.9 \%)$ & $19(3.1 \%)$ & $2(1.7 \%)$ \\
Burn wounds* & $18(2.5 \%)$ & $11(1.8 \%)$ & $7(5.8 \%)$ \\
\hline
\end{tabular}

${ }^{*} P$ value $<0.05$.

TABLE 2: Transport characteristics.

\begin{tabular}{lcc}
\hline & $\begin{array}{c}\text { Transport } \\
\text { method }\end{array}$ & $\begin{array}{c}\text { Mean }(95 \% \text { confidence } \\
\text { interval) }\end{array}$ \\
\hline $\begin{array}{l}\text { Relative transport } \\
\text { Time }^{\circledR *}\end{array}$ & Ambulance & $79(77.1-80.3)$ \\
\hline $\begin{array}{l}\text { Distance in km } \\
\text { (road) }\end{array}$ & AmbMS & $39(37.1-41.3)$ \\
\hline $\begin{array}{l}\text { Average speed in } \\
\text { km/h* }\end{array}$ & HEMS & $29(27.6-30.0)$ \\
\hline $\begin{array}{l}\text { Average time benefit } \\
\text { in minutes* }\end{array}$ & Ambulance & $79(76.8-81.1)$ \\
\hline
\end{tabular}

${ }^{*}$ Results differ significantly $(P<0.001)$ between ambulance and HEMS

${ }^{\circledR}$ Relative transport time was calculated relative to the transport time predicted by Google Maps.

TABLE 3: Mean relative transport time by patient characteristics.

\begin{tabular}{lcccccc}
\hline \multirow{2}{*}{ Trauma mechanism } & \multicolumn{3}{c}{ Ambulance } & \multicolumn{3}{c}{ HEMS } \\
& No* & Yes* & $P$ value & No* & Yes* & $P$ value \\
\hline High energy trauma & 79 & 79 & 0.67 & 41 & 39 & 0.43 \\
Traumatic brain injury & 79 & 78 & 0.35 & 40 & 39 & 0.55 \\
Intubation & 78 & 79 & 0.38 & 49 & 38 & 0.15 \\
Resuscitation & 78 & 84 & 0.03 & 39 & 40 & 0.85 \\
Child & 78 & 81 & 0.31 & 39 & 39 & 0.89 \\
Burn wounds & 79 & 84 & 0.33 & 39 & 47 & 0.06 \\
Drowning & 79 & 82 & 0.42 & 39 & 39 & 0.99 \\
\hline
\end{tabular}

*Values represent the relative transport time.

In Table 3 the relative transport time is shown for the presence and absence of the different patient and trauma variables. Ground transport of resuscitated patients seemed somewhat faster compared to HEMS transport, but the difference is small and only borderline significant. None of the other trauma mechanism variables had a significant different transport time, both in ambulance and HEMS transport.

Also, none of the external influences we tested (snow, frost, night time, and wind direction) showed significant impact on transport time. We also tried to show the effect of nearby or distant landing facilities on average transport time, but there were only 6 air transports to hospitals with distant landing facilities, making adequate analysis impossible.

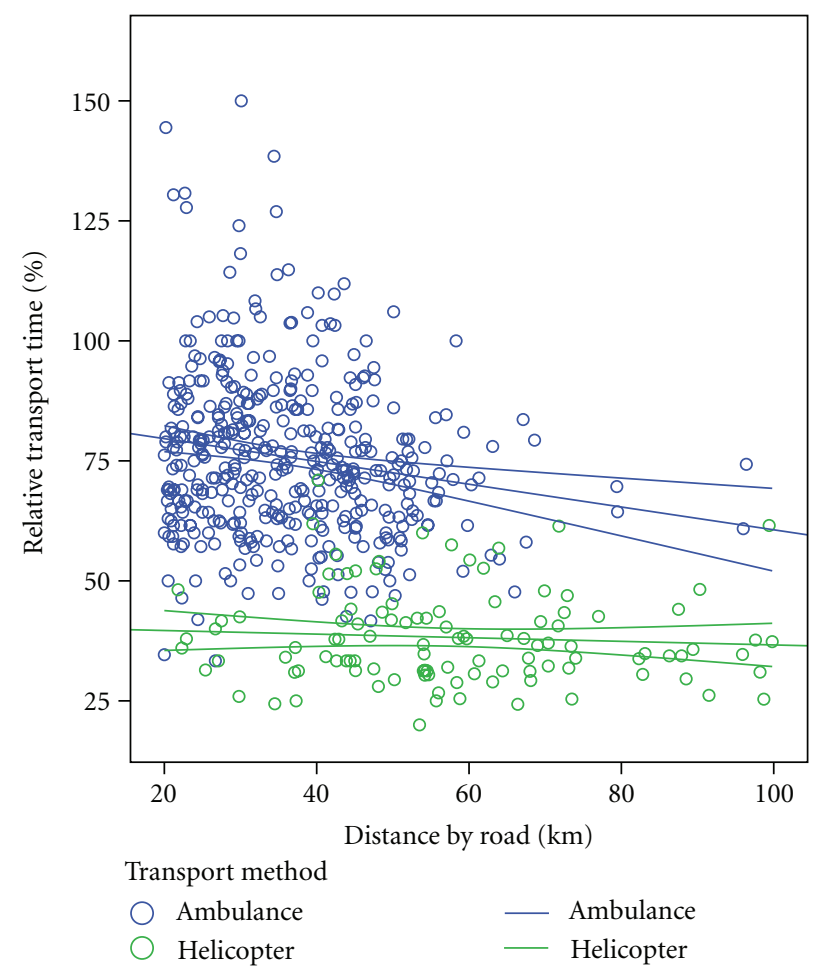

FIGURE 1: Scatter plot with linear regression lines per transport method. Average relative transport time per distance for both HEMS helicopter and ambulance with linear regression line.

The effect of distance on relative transport time is shown in Figure 1. This figure shows a slightly decreasing line (with 95\% CI) for the ambulance, and a near-horizontal line for the helicopter. Both lines do not cross. This clearly shows that even at short distance, air transport is on average much faster than ground transport. The average relative transport time of an ambulance is 79\% (95\% confidence interval (CI) $77.1-80.3)$ and helicopter transport averages at 39\% (95\% CI 37.4-42.9).

There was no helicopter transport at a distance less than $20 \mathrm{~km}$, so we have no data from the HEMS at short distance, but overall in this dataset helicopter transport is nearly twice as fast as ambulance transport. This is consistent with Figure 2, which shows average minutes of time benefit per distance with $95 \%$ CI. This also clearly shows an average larger absolute time benefit for the helicopter compared to the ambulance.

\section{Discussion}

4.1. Main Results. The main question in this study was: what factors contribute to a faster or slower helicopter transport, and when is it faster to fly and when is it faster to drive to the hospital. Our hypothesis was that the helicopter is faster at longer distance than the ambulance, because of the higher maximum speed and the straight line that can be followed, but it is slower at shorter distance due to taking off and landing procedures. However, the results of this study show that even on short distance, air transport by HEMS 


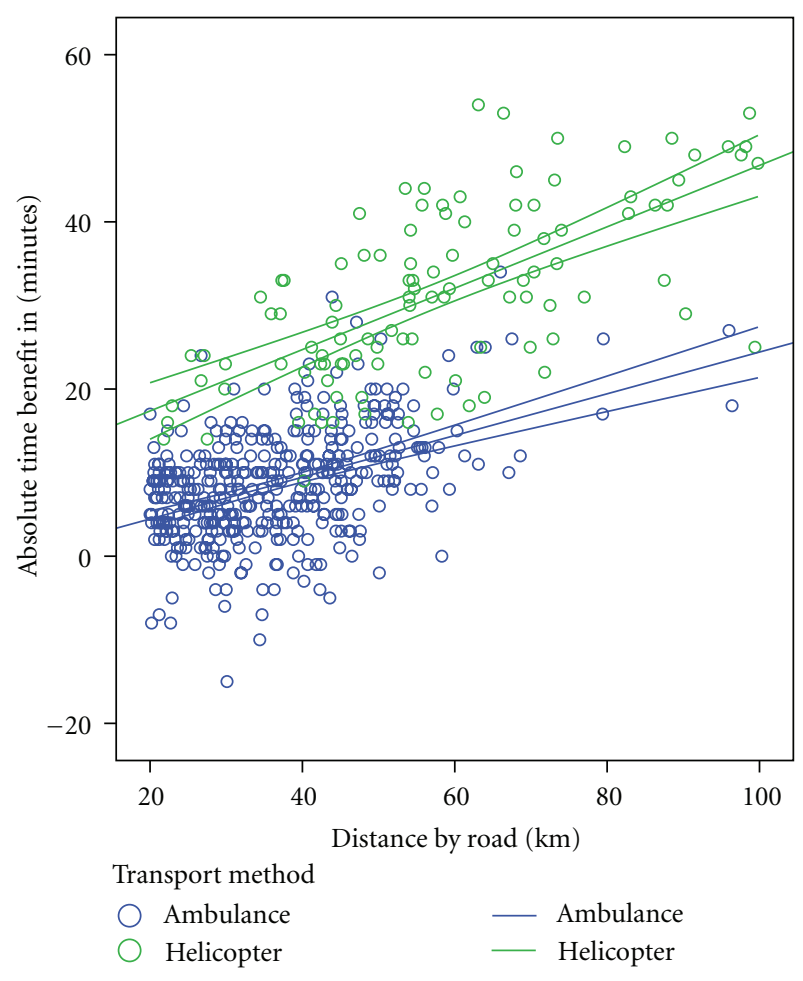

FIGURE 2: Scatter plot with absolute minutes of time benefit per distance with linear regression lines. Absolute time benefit is defined as actual transport time minus predicted transport time (by Google Maps).

was nearly always faster, independent of trauma mechanism. The exact threshold when it is faster to transport by HEMS cannot be determined with this data, because we have no HEMS transport data below 20 kilometres. We are aware that local infrastructure, density of hospitals, and availability of landing facilities each have impact on transport time. However, a smaller study on this subject performed in the starting years of the Nijmegen HEMS shows equal results, which are even significant on distances starting from 10 kilometres [6]. Given the consistency of the data shown in Figure 1, the similar results in the aforementioned study, and the nature of helicopter transport, we believe the calculated advantage on transport time to be accurate and that it likely applies for other regions in the Netherlands.

Another point of discussion is that the relative transport time (RTT) decreases for the ambulance with distance more than for the helicopter, so in theory the ambulance would be faster on even longer distances than the helicopter. This is theoretically impossible since the top speed for the helicopter is much higher than that of an ambulance. The decrease in ambulance RTT with distance only shows that distance has a larger impact on the ambulance transport time, whereas the helicopter has reached a constant peak velocity at 20 kilometres, keeping the RTT stable. Also, we used Google Maps to calculate a target time. Although we do not know what algorithms are used to produce the target times, we can safely assume the results are at least consistent. Even if the target times are not accurate, the relative difference between ambulance and helicopter is still valid.

The average absolute time benefit for the ambulance was 7 minutes, and for the helicopter it was 31 minutes. However, the helicopter is used on significantly longer distance while the ambulance is mainly used for short distance, so the absolute time benefit should be calculated per distance, as is shown in Figure 2.

The transport times of the HEMS helicopter include unloading and transport to the ER for the heliport. However, it does not include loading the patient in the HEMS helicopter. This time can be substantial when the helicopter had landed at distance from the accident, and should be taken in consideration for each transport individually. Another point of discussion is that the retrospectively found weather reports are averages of that day, and there is no guarantee that these are representative of the actual conditions the HEMS faced at the moment of scramble. Further study with more accurate weather descriptions is needed to show the influence of the weather conditions on transport time for both ground and air transport.

Finally, we only studied transport time, and did not look at outcome, for example, did faster transport really lead to less morbidity and less mortality? We have found quite some literature that shows helicopter transport to reduce mortality in trauma patients [7-10], and although this was not within the scope of this study, we believe that our results combined with this literature justify a recommendation to use transport by the HEMS helicopter more often in the Netherlands rural setting.

4.2. Limitations. This study has some limitations. This is a retrospective study, which has not been randomized. However, a double blind study is impossible and a randomized study is highly impractical.

We may have some missing or inaccurate data, which cannot be prevented given the setup of this study. We rely on accurate administration by the HEMS doctors. Because the HEMS crew is required to register landing locations very precisely, we trust that this administration is accurate. Weather data are averages of that day for each region and cannot be a fully accurate representation.

\section{Conclusion}

With present data, we have shown that trauma mechanism, injury severity, and weather influence do not influence the transport time of patients from the scene of accident to the hospital for both HEMS helicopter and ground ambulance transport. However, our results clearly show the enormous advantage of the HEMS air transport on transport time, compared to transport by ground ambulance in the Netherlands. For the ambulance, the transport time averages at $79 \%$ of predicted transport time by Google Maps, for the HEMS this transport time is 39\%: nearly twice as fast.

We strongly recommend good landing facilities at receiving hospitals to make optimal use of this fast method of transportation. The time benefit over ambulance transport can be lost when inadequate landing facilities force distant 
landing. For optimisation of helicopter transport times to specialized trauma centres, heliports near the emergency department are essential.

\section{References}

[1] H. van Schuppen and J. Bierens, "Understanding the prehospital physician controversy. Step 1: comparing competencies of ambulance nurses and prehospital physicians," European Journal of Emergency Medicine, vol. 18, no. 6, pp. 322-327, 2011.

[2] http://www.zorgatlas.nl/zorg/ziekenhuiszorg/traumazorgenen/ reistijd-tot-dichtstbijzijnde-traumacentrum-2009.

[3] M. A. Diaz, G. W. Hendey, and H. G. Bivins, "When is the helicopter faster? A comparison of helicopter and ground ambulance transport times," Journal of Trauma, vol. 58, no. 1, pp. 148-153, 2005.

[4] P. J. Karanicolas, P. Bhatia, J. Williamson et al., "The fastest route between two points is not always a straight line: an analysis of air and land transfer of nonpenetrating trauma patients," Journal of Trauma, vol. 61, no. 2, pp. 396-403, 2006.

[5] K. E. Stewart, L. D. Cowan, D. M. Thompson, and J. C. Sacra, "Factors at the scene of injury associated with air versus ground transport to definitive care in a state with a large rural population," Prehospital Emergency Care, vol. 15, no. 2, pp. 193-202, 2011.

[6] C. H. T. Tan, M. D. Sprockel, and J. Biert, "Transport of trauma victims-by ground or air?" Waypoint AirMed and Rescue, no. 31, August-September 2008.

[7] E. E. Sullivent, M. Faul, and M. M. Wald, "Reduced mortality in injured adults transported by helicopter emergency medical services," Prehospital Emergency Care, vol. 15, no. 3, pp. 295302, 2011.

[8] J. B. Brown, N. A. Stassen, P. E. Bankey, A. T. Sangosanya, J. D. Cheng, and M. L. Gestring, "Helicopters improve survival in seriously injured patients requiring interfacility transfer for definitive care," Journal of Trauma, vol. 70, no. 2, pp. 310-314, 2011.

[9] J. McVey, D. A. Petrie, and J. M. Tallon, "Air versus ground transport of the major trauma patient: a natural experiment," Prehospital Emergency Care, vol. 14, no. 1, pp. 45-50, 2010.

[10] A. D. Mitchell, J. M. Tallon, and B. Sealy, "Air versus ground transport of major trauma patients to a tertiary trauma centre: a province-wide comparison using TRISS analysis," Canadian Journal of Surgery, vol. 50, no. 2, pp. 129-133, 2007. 


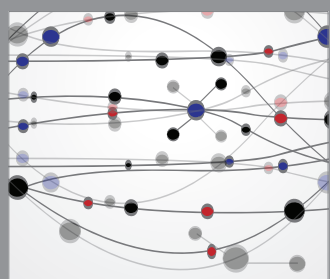

The Scientific World Journal
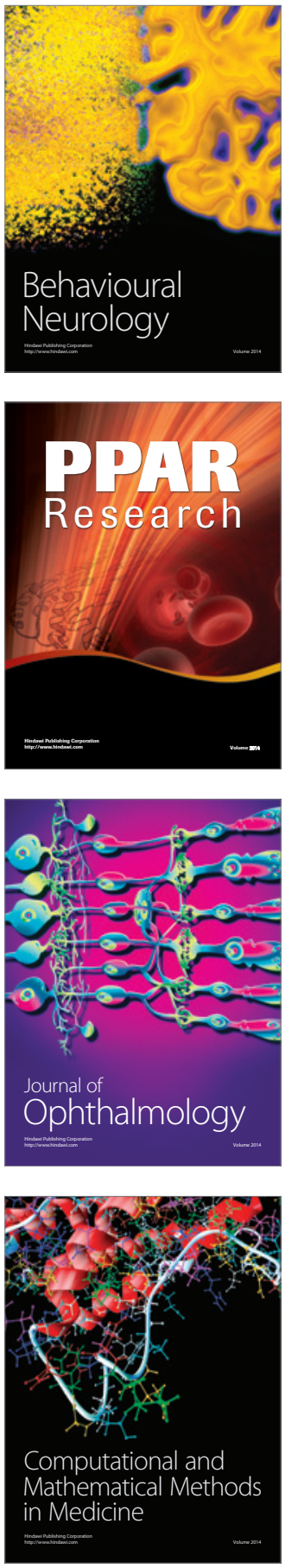

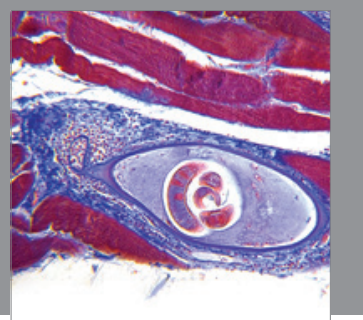

Gastroenterology

Research and Practice
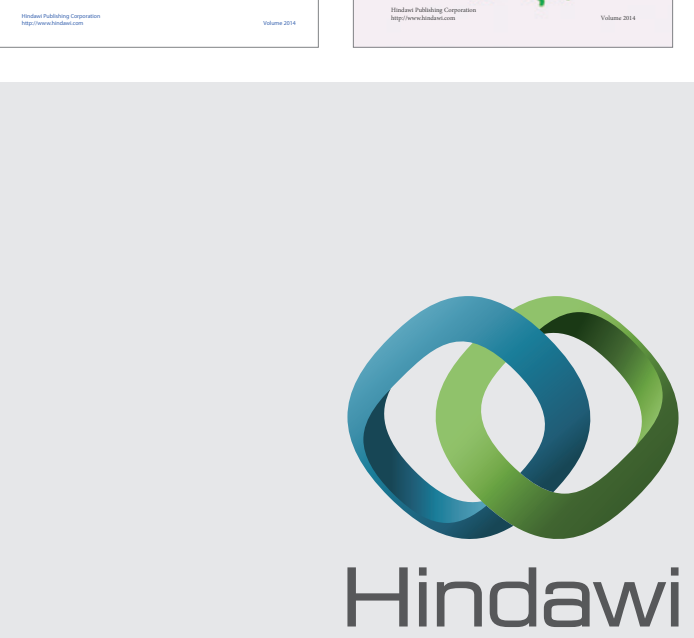

Submit your manuscripts at

http://www.hindawi.com
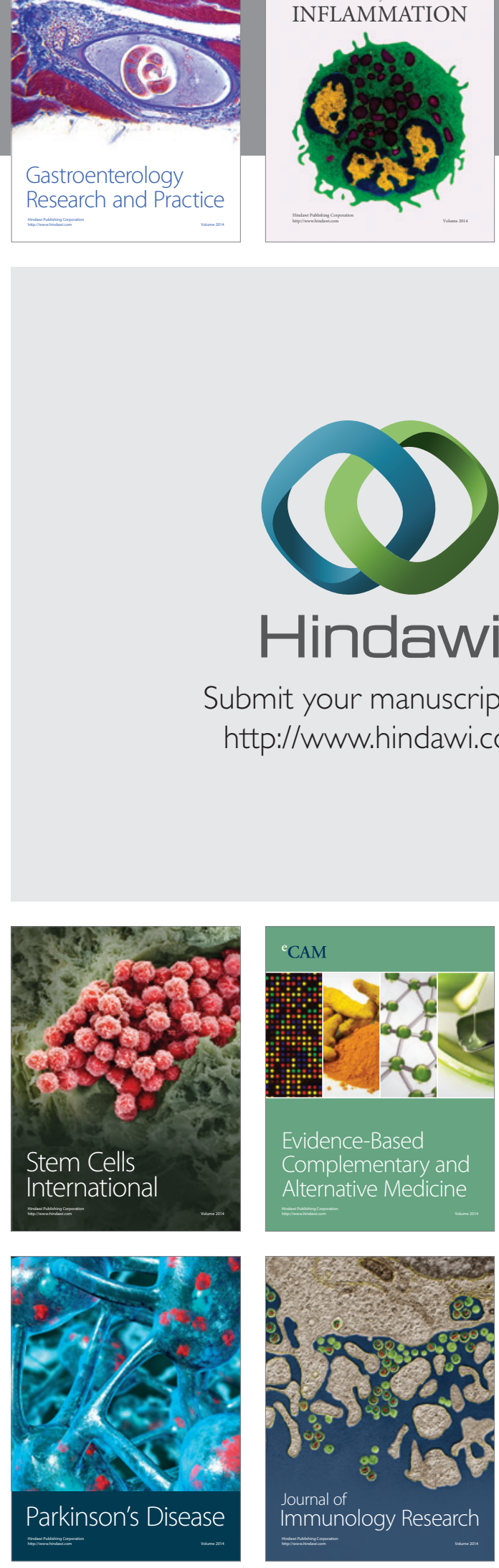

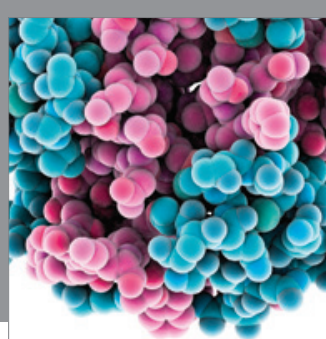

Diabetes Research
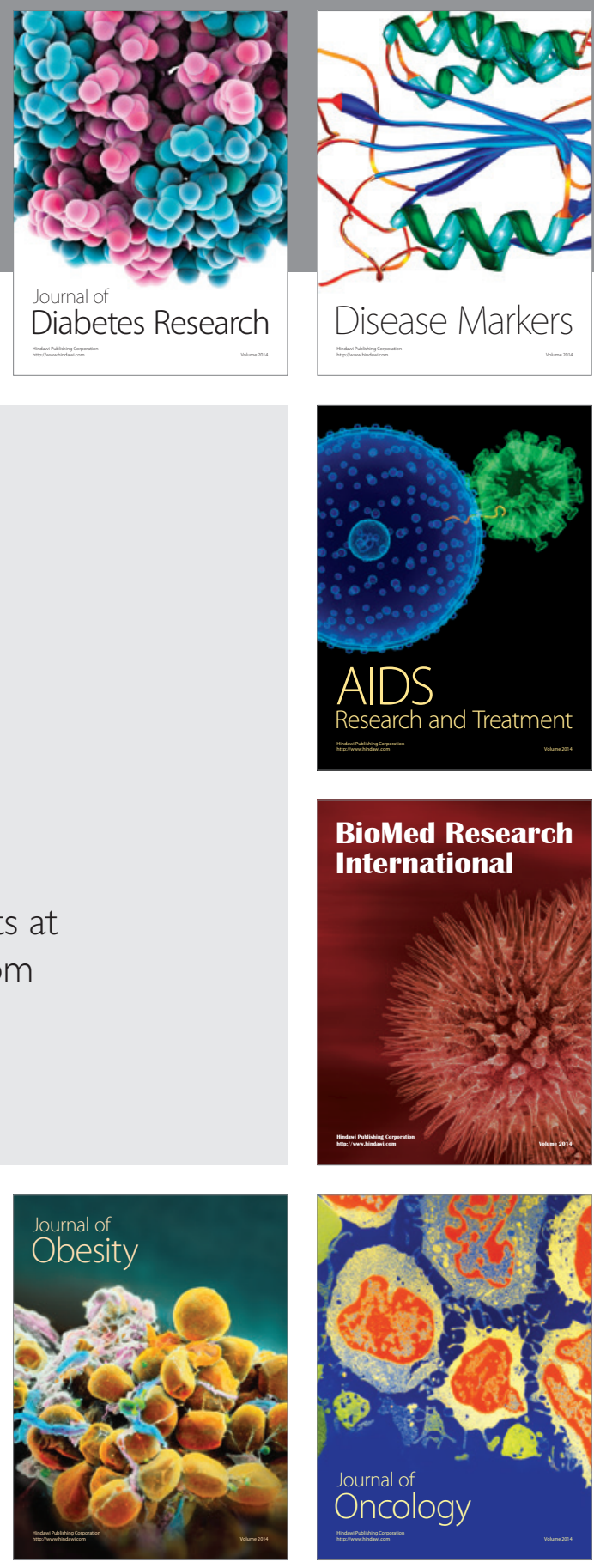

Disease Markers

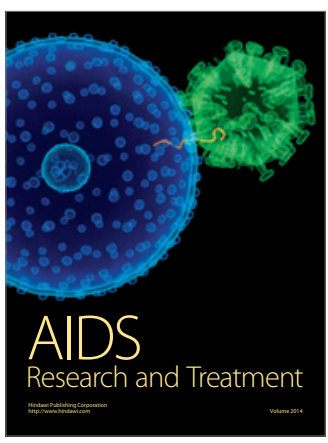

BioMed Research

International
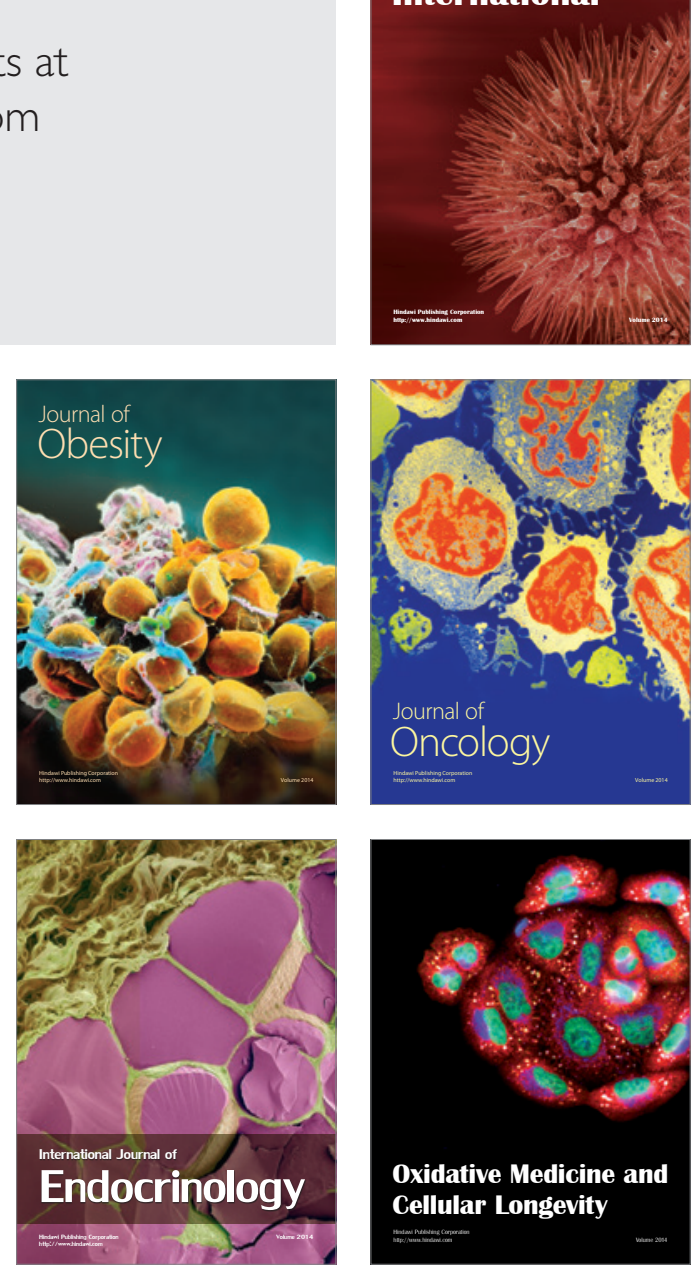